\title{
Wie tönen die Tschechen ihre Aussagen ab? \\ Eine Auseinandersetzung mit Abtönungskonstruk- tionen im (gesprochenen) Tschechischen und ihrer Übersetzung ins Deutsche
}

\section{How Do Czechs Modify Their Speech? Examining the Constructions of Propositional Modification in (Spoken) Czech and Their Adequate Translation into German}

Jan Ciosk

\begin{abstract}
This article deals with several constructions used for propositional modification (in German "Abtönung") in Czech and aims to suggest an adequate translation into German. As part of the theoretical framework, crucial functional and formal characteristics of this phenomenon are addressed together with methods of testing the implicit content of the given construction. The structural analysis concentrates on both lexical and morpho-syntactical manifestations of the phenomenon. Firstly, the particle "jako" and its communicative functions are presented, then we focus on processes that operate on higher levels of the Czech language. These include the process of framing or tag questions.
\end{abstract}

\section{Keywords}

propositional modification; modal particles; Abtönung; intentionality; metalinguistics; contrastive linguistics; translation; 


\section{Einleitung}

Im Fokus dieses Aufsatzes steht das Phänomen der Abtönung, die als eine der wichtigen Komponenten sowohl der gesprochenen als auch der geschriebenen Sprache zu betrachten ist. Die Auseinandersetzung mit dieser Erscheinung hat im deutschen Sprachraum bereits eine längere Tradition, im Rahmen der tschechischen Linguistik ist der Begriff eher wenig bekannt, und das Phänomen wird nur teilweise oder auf Umwegen behandelt.

Die ersten zielgerichteten Arbeiten, die auf Deutsch geschrieben wurden und die sich den modifizierenden Funktionen einiger Lexeme widmeten, entstanden in den späten 1960er Jahren. Wir möchten dabei vor allem die Beiträge von Weydt (1969) und Helbig (1987) nennen. Weydt und seine Nachfolger haben sich seit dem Ende der 1960er Jahre bemüht, das linguistische Repertoire, das man zur sprecherbezogenen Modifizierung der Aussage verwendet, systematisch zu beschreiben. Die Mehrheit konzentrierte sich dabei auf die Erforschung der sog. Abtönungspartikeln (oder auch APn). Exemplarisch können wir etwa die komparative Studie zu deutschen und englischen APn von Bublitz (1978) oder die Monografie zu Partikeln und ihrer Kombinatorik von Thurmair (1989) nennen. Die Kategorie der APn und zum Teil auch das Phänomen Abtönung selbst ist dann in den meistbenutzten Grammatiken und ähnlichen Quellen (etwa DUDEN, grammis) unter dem entsprechenden Stichwort zu finden. Man findet auch in älterer Literatur Bemerkungen zu der mehrdeutigen Natur von Wörtern wie $j a$ oder $d o c h,{ }^{1}$ intensiver setzt man sich mit ihnen jedoch etwa seit der pragmatischen Wende auseinander.

Tschechische Forscher haben das Thema bislang bis auf Ausnahmen eher vernachlässigt. Wir möchten vor allem zwei Monografien nennen, in denen sich die Autoren ausführlicher mit der Beschreibung einzelner APn (konfrontativ in beiden Sprachen) und somit auch mit der Bestimmung der kommunikativen Eigenschaften des Phänomens befassen. Nekula (1996) widmet sich neben der systematischen Einordnung einzelner Partikeln auch der Analyse ihrer Ähnlichkeit mit den deutschen Entsprechungen. Eine umfassende Analyse der APn doch und ja und ihre Anknüpfung an die tschechischen Äquivalente finden wir bei Rinas (2006b). Außer Nekula und Rinas findet man Bemerkungen zur Abtönung nur selten, am häufigsten im Rahmen vereinzelter Analysen. Grepl \& Karlík (1998) beschäftigen sich mit dem Prozess der Aussagemodifikation (výpovědni modifikace), die man mit Bezug auf die funktionalen Eigenschaften als eine passende Übersetzung des Ausdrucks Abtönung ansehen kann. Sie gelangen zu einer Teildefinition, dass

„manche Aussagemodifikationen in Form fester Konstruktionen vorkommen, die für verschiedene Funktionen konventionalisiert sind, z. B. für die Äußerung bestimmter kommunikativer Ziele und Einstellungen, und die (oft gleichzeitig) ein wichtiges Mittel der Textkohäsion und -kohärenz sind.“2 (Grepl \& Karlík 1998:389)

1 Vgl. dazu die Überlegungen von Gottsched (1758) oder Heynatz (1796). Vgl. hierzu auch Rinas (2011: 99-104).

2 Im Original: "některé výpovědní modifikáty nabývají povahy ustálených syntaktických útvarů, konvencia- 
Eine Auseinandersetzung mit den erwünschten kommunikativen Zielen, die wir unter Abtönung einordnen können, finden wir auch in der tschechischen Akademiegrammatik Mluvnice čěstiny (1986). Wir möchten hier vor allem auf die Liste diverser Arten von Partikeln hinweisen, die in diesem Handbuch analysiert und summiert werden.

Wir werden uns im Folgenden bemühen, aufgrund mehrerer Ansichten das Konzept der Abtönung zu behandeln und ihre grundlegenden Komponenten zu definieren. Den theoretischen Abhandlungen folgt ein analytischer Teil, in dem wir zeigen möchten, welche Konstruktionen im Tschechischen vorkommen können, wenn sich der Sprecher entscheidet, die Aussage abzutönen. Alle Beispiele und ihre Übersetzungen, die im Folgenden genannt werden, sind Teile des vom Autor erstellten Mikrokorpus und wurden von einer kleineren Kontrollgruppe (10 Probanden) geprüft. Es handelt sich dementsprechend um Übersetzungsvorschläge, die vor allem als Veranschaulichung dienen und eine von mehreren für die Situation adäquaten Varianten darstellen.

\section{Begriffsklärung}

Bei der Erfassung der linguistischen und pragmatischen Besonderheiten dieses Phänomens müssen gleich mehrere Aspekte in Betracht gezogen werden. Wir beginnen mit der Bedeutungsanalyse von Aussagen, die über eine abtönungskonstituierende (lexikalische) Einheit verfügen. Die Analyse des Satzmodus, der von den Einzelteilen der Aussage gebildet wird, bringt uns nicht zu ihrer aktuellen Bedeutungstragweite bzw. reicht nicht für die Bestimmung aller Absichten, die der Sprecher in eine solche Aussage eingebettet hat. Zwar steht der Satzmodus gewissermaßen zwischen der konsolidierten Satzbedeutung und dem illokutiven Akt, den der Sprecher verwenden will, es wäre aber präziser zu behaupten, dass er mithilfe der abtönungsfähigen Mittel eher modifiziert wird (vgl. Lang 1988; Meibauer 1994). Eine Zuordnung zu einem einzigen Sprechakt wäre dann beispielsweise bei manchen Lexikoneinheiten wie die APn tak oder doch eher schwierig.

\section{(1) Tak neplač! \\ Wein doch nicht!}

In dem genannten Beispiel (1) erfüllt der Ausdruck tak bzw. doch gleich mehrere Funktionen. Im Vergleich zu dem reinen Imperativsatz, den man nur mithilfe des Verbs bilden würde, fügt der Sprecher weitere äußerungsspezifische Informationen hinzu. Die befehlende Natur der Aussage wird weiter modifiziert, dem Illokutionstyp (in diesem Falle geht es um eine Direktive) ${ }^{3}$ werden weitere Unterarten bzw. Abstufungen

\footnotetext{
lizovaných pro různé funkce, např. pro vyjadřování jistých komunikačních cílů, postojů a jsou (často zároveň) důležitým prostředkem textové návaznosti, soudržnosti."

3 In diesem Aufsatz werden wir bei der Analyse der illokutiven Funktionen von abtönungsfähigen Konstruktionen unter anderem auf die theoretischen Grundlagen der Sprechakttheorie von Austin (1972) und Searle (1969) hinweisen.
} 
zugeschrieben. Man könnte dann dementsprechend die Aussage als eine (tröstende) Bitte oder ein (entschiedenes) Verbot interpretieren. Die kommunizierten Nuancen projizieren sich in die gegebene Abtönungskonstruktion, in diesem Falle in eine AP, hinein und stellen somit ein Mittel dar, das zur weiteren Präzisierung der Formulierung von (impliziten) Sprecherintentionen beiträgt. Waltereit (2006: 25f) nennt diese Prozesse „illokutionäre Spezifikationen“ und will damit zum Ausdruck bringen, dass sich der Bedeutungskern der Aussage nicht ändert, wenn ihr auch der Sprecher zusätzliche Elemente beifügen möchte.

Die kleinen Unterschiede, die wir normalerweise nur schwer aus einem Beispiel wie (1) ablesen würden, hängen vom situativen Kontext ab. In neueren Studien wird dieser Bezugspunkt auch „pragmatischer Prätext“ genannt (vgl. Fischer 2000; Diewald 1999). Die Gesprächspartner kommunizieren miteinander auf zwei Ebenen, und das Kontextabhängige wird in der Formulierung einigermaßen versteckt. Der propositionale Gehalt der Aussage unterscheidet sich von dem nicht-propositionalen in mehrfacher Hinsicht. Beispielsweise operiert man auf der propositionalen Ebene mit Denotaten, also mit dem begrifflichen Kern des Ausdrucks, deren Gesamtheit dann eine Proposition (einen Aussageinhalt) bildet. Eine wichtige Eigenschaft einer solchen Proposition ist die Feststellbarkeit ihrer Wahrheitswerte.

(2) Helga jde domů.

Helga geht nach Hause.

(3) Helgo, jdi domů!

Helga, geh nach Hause!

Die unter (2) angeführte Proposition beinhaltet genug Informationen, um einen Aussageinhalt zu bestimmen. Es handelt sich um einen Aussagesatz, dessen Funktion es ist, den Hörer zu informieren, ihm etwas mitzuteilen. Wenn wir dessen Aussageinhalt mit dem Inhalt in (3) vergleichen, kommen wir zu demselben Sachverhalt. Der Unterschied bildet die Intention des Sprechers. Während sich der Sprecher in (1) für eine bloße Mitteilung entschieden hat, fordert der Sprecher in (3) den Hörer zu einer Tat auf. Was beabsichtigt wird, erkennen wir an der illokutiven Funktion der Äußerung, einer Aufforderung. Prozesse, die die Absichten des Sprechers, Verweise auf das gemeinsame Wissen und den Kontext, finden auf der nicht-propositionalen Ebene statt, und deshalb ist dies auch der Wirkungsbereich der Abtönung.

Nekula (1996:45-47) betont unter anderem auch die Polyfunktionalität und Kombinierbarkeit einiger abtönungsfähigen Mittel. Ihre Verwendungsweisen in bestimmten Satztypen zeigen, dass sie eine für die Äußerung spezifische Rolle spielen, indem sie in verschiedenen Satztypen verschiedene Illokutionen begleiten und verstärken können.

(4) Petře, měli jsme domácí úkol!

To jsem já ale nevěděl.

Petr, wir hatten eine Hausaufgabe.

Das wusste ich ja/aber nicht. 
(5) (Tak) ale pojd' už, ujede nám autobus!

(So) komm doch (endlich mal), wir verpassen den Bus!

Die AP ale wird in den obengenannten tschechischen Beispielen auf zweierlei Art und Weise zum Ausdruck gebracht, jedes Mal im Rahmen eines anderen Illokutionstyps und eines anderen Satztyps. In (4) kommt sie in einem Aussagesatz vor, in dem sie von Seiten des Sprechers eine Rechtfertigung zum Ausdruck bringen soll. Mithilfe der AP wird in der Aussage die Tat (in unserem Falle eher die Untätigkeit) des Sprechers begründet. Brausse (1986) setzt sich mit den argumentativen Charakteristika bei der AP ja auseinander und behauptet:

„Nicht immer sind natürlich im Gespräch These und Argument explizit ausgedrückt. Es ist für Alltagssprache charakteristisch, dass nur ein Teil dessen, was für das Verständnis des Dialogs notwendig ist, auch ausdrücklich ausgesprochen wird. So ist es auch in Argumentationen. [...] Es ist die Regel, dass Prämissen und/oder Schlussfolgerungen nicht explizit gemacht, aber mitverstanden werden." (S. 214)

Die implizite Argumentation, die die Aussage unterstellt, verstärkt die pragmatische Funktion des Satzes. Es handelt sich um eine Assertive, die in einen Aussagesatz eingebettet ist. In (2) hingegen, sehen wir eine Verwendungsweise von ale in Form eines Befehls, einer Bitte oder etwa einer nachdrücklichen Einladung. Der Ausrufesatz, und die daraus gehende Direktive, wird mithilfe der AP modifiziert (ähnlich wie in unserem Beispiel (1)). Der Wirkungsskopus der APn und ähnlicher Konstruktionen ist dementsprechend auf diejenigen Kontexte beschränkt, die ihnen der Satzmodus oder Satztyp der entsprechenden Aussage erlaubt. Die Interpretation der nicht-propositionalen und vor allem recht polyfunktionalen Wirkung der Abtönung muss also ipso facto auf der propositionalen Basis gegründet werden. Dies ist Nekula (1996:46) zufolge eine der wichtigsten Eigenschaften der Abtönung, die sie in Opposition, beispielsweise zu Subjunktionen stellt, vergleiche etwa:

(6) Mlčí. - At̉ mlčí!

Er schweigt. - Er soll schweigen!

Während die APn ale in (5) oder tak in (1) einer weiteren (kontextabhängigen) Spezifizierung im Rahmen einer direktiven Aussage dienen, lässt sich dasselbe nicht für die in (6) genannte Subjunktion behaupten. Wenn der Sprecher nämlich dieses Lexem dem ursprünglich rein informativen Einzelsatz beifügt, ändert sich grundsätzlich seine Bedeutung und Wirkung. Von einer Assertive zu einer Direktive. Einen solchen Wechsel sehen wir beispielweise in (1) nicht. 


\section{3 Überprüfung des Abtönungsinhalts}

Das Inventar der APn und ähnlich wirkender Mittel ist im Tschechischen nicht so groß wie etwa im Deutschen oder Niederländischen. Da wir für unsere Zwecke nicht nur eine Analyse von einzelnen Lexemen durchführen werden, sondern uns mit der Existenz der Abtönung auch auf anderen Ebenen der tschechischen Sprache befassen möchten, setzen wir uns im Folgenden kurz mit ausgewählten Methoden der Überprüfung des Abtönungsinhalts auseinander. Aus dem Spektrum der Herangehensweisen haben wir uns entschieden, zwei vorzustellen.

Mit dem Gebrauch von APn wird die Aussage kommentiert und dem Hörer ein Signal gesendet. Da die Kommentare nicht offen (i. e. in Form eines in semantischer Sicht nicht vollständig formulierten Satzes bzw. Satzteils) ausgedrückt werden, sprechen wir von Metakommentaren. Diese Fähigkeit ist nicht auf die ganze Klasse der APn applizierbar; eine Mehrheit ist aber klar als eine Gruppe von Ausdrücken zu definieren, die zur Äußerung von epistemischen Einstellungen des Sprechers dient (vgl. König [2010]). Für die Entschlüsselung der versteckten subjektiven Einschätzungen, Wertungen und anderer sprecherspezifischer Informationen werden mehrere Methoden verwendet. Diese fungieren dann als Mittel zur Rechtfertigung oder eben Ablehnung des entsprechenden Übersetzungsvorschlags, weil man auf ihrer Grundlage die kommunizierte Information im Ausgangstext und die in den Zieltext übertragene Formulierung vergleichen und kritisch analysieren kann.

Die wohl meistverwendete Methode, nicht nur für die Analyse der abtönungshaltigen Ausdrücke beim Übersetzen, ist die Deletionsmethode. Die Bestimmung von kommunikativen Werten der Aussage mit und ohne AP (oder einer anderen Konstruktion mit derselben Wirkung) besteht auf dem bloßen Vergleich zweier Möglichkeiten, z. B.:

(7) Louvre se nachází v Pařízii.

Louvre se préce nachází v Paříži.

Der Louvre befindet sich in Paris.

Der Louvre befindet sich doch in Paris.

Diese Methode empfiehlt sich für eine grobe Bestimmung der Funktion von der jeweiligen AP/Konstruktion, sie ist jedoch nicht für eine detaillierte Analyse aller Bedeutungsvariierungen geeignet. Die Komplexität der Ausdrucksmöglichkeiten (und dementsprechend auch der Übersetzungsmöglichkeiten) von doch erlaubt ohne Kontext und weiteres Einsetzen in die entsprechende Gesprächssituation keine Schlussfolgerungen zu ziehen (vgl. Rinas (2006b)).

Als zweite Möglichkeit möchten wir die Methode des sog. Nachsatzes nennen. In einem solchen Untersuchungsvorgang wird der Aussage, die eine abtönungskonstituierende Konstruktion beinhaltet, ein Nachsatz beigefügt, der diese näher bestimmt und hervorhebt. Oft bildet dieser Satz eine Opposition zu dem, was im vorangegangenen Satz, beispielsweise in Form einer AP, geäußert wird, vgl.: 
(8) Já to nebyl!

Kdo to tedy byl? (když ne ty)

Das war ich nicht!

Wer also war es? (wenn nicht du)

(9) V̌sak on přijde. (nemusíš si dělat starosti)

Er kommt ja. (du musst dir keine Sorgen machen)

In beiden oben genannten Beispielen sehen wir, dass sich der im Nachsatz genannte Inhalt im vorangegangenen Satz klar mithilfe der APn tedy/also und však/ja manifestiert. Die Widerspiegelung wird jedoch nur dann deutlicher, wenn man dann zusätzlich die Methode der Deletion verwendet. Erst mit der Kombination der zwei genannten Methoden ist es möglich, die tatsächliche kommunikative Funktion und ihre Einbettung im Aussagetext zu finden und bei der Übersetzung zu übernehmen. Beide Methoden sind jedoch nicht für alle Abtönungsmittel anwendbar (dazu etwa Bastert [1985: 25ff]).

\section{Abtönungsarten}

Wir möchten im Folgenden einzelne Äußerungsarten behandeln, die im Tschechischen beim Prozess der Abtönung verwendet werden. Im Fokus werden nicht nur lexikalische Erscheinungen stehen, sondern auch morphosyntaktische und intonatorische Sprachelemente.

Zuerst widmen wir uns der (in beiden Sprachen) meistvertretenen Kategorie der abtönungskonstituierenden Mittel, nämlich den APn. Die grundlegenden Charakteristika und einen Abriss der wichtigsten funktionalen und formalen Eigenschaften finden wir bereits bei WEYDT (1969) und (1977). Nennenswert für unsere Zwecke sind vor allem die folgenden Punkte:
(a) APn sind nicht flektierbar
(b) APn sind meist unbetont
(c) APn sind nicht koordinierbar
(d) APn haben Skopus über den ganzen Satz

Thurmair (1989) trägt zur deutschen Partikelforschung mit einem Werk über dessen gegenseitigen Kombinatorik bei und erweitert somit das Spektrum der Analyse nicht nur für Übersetzungswissenschaftler. Außer Nekula (1996) finden wir in der tschechischen Fachliteratur Überlegungen zm Verhältnis der tschechischen und deutschen APn bei Masařík (1982), der die These vertrat, dass die tschechischen APn zu den deutschen eine besondere Parallelität aufweisen, sowohl im Rahmen der kommunikativen Kompetenzen als auch im Bereich ihrer Distribution. Man kann sagen, dass damit die Grundlage für weitere Auseinandersetzungen gebildet wurde, wie etwa bei Spěváková (1992). 
Wir wenden uns jetzt der AP jako zu. In der gesprochenen Sprache kann man im Tschechischen das Wort jako entweder als ein reines Füllwort finden oder mit besonderer metakommunikativer Funktion. Die erste Variante führen wir unter (10) an.

(10) Ona je jako... svobodná? A to jako... s nikým jako nežije?

Sie ist (also) ... ledig? Und da (also) ... lebt sie (also) ... mit niemandem zusammen?

Kolářová (1994:168) bemerkt, dass die unter (10) angeführte Verwendungsweise von jako darauf hinweist, dass der Sprecher über die Aussage (oder genauer über die passende Formulierung) ad hoc nachdenkt oder aber dass er sich nicht sicher ist. Vgl. dieses Beispiel mit den folgenden Mikrotexten:

(11) Anděla chce tvoje telefonní číslo.

Proč bych jí ho jako měla dávat?

Anděla will deine Telefonnummer.

Warum sollte ich sie ihr denn (bitte) geben?

(12) Oni to opravdu nevěděli.

Já to jako chápu, ale mohli napsat.

Sie wussten es wirklich nicht.

Ich verstehe das ja auch, aber sie konnten schreiben.

Für das Beispiel (11) gilt, dass die AP in Kontexten verwendet wird, in denen viel argumentiert wird und wo man oft Spannungen oder eine Art Konflikt erwarten kann. Mithilfe der abgetönten Aussage (in den meisten Fällen handelt es sich um eine Frage) will der Sprecher, der sich in einer problematischen Situation befindet, eine Lösung finden. Rinas (2013:171) vertritt die These, dass der Fragende mit dem Gebrauch präsuponniert, dass der Befragte eine Lösung anbieten kann oder sogar anbieten muss, weil er für die Entstehung der Situation verantwortlich ist. Gleichzeitig sind in der Aussage Verwunderung und eine mögliche Übertragung der Beweisführung auf den momentanen Gesprächspartner eingebettet. In (12) kommen wir zu einem anderen Kontext, in dem jako vorkommt. Die Vergewisserung, die von dem Sprecher angeboten wird, stellt allerdings noch mehr dar. In einem gewissen Sinne können wir von einem Wissensvorsprung ausgehen, da die mit jako angedeutete Berücksichtigung des vorangestellten Arguments später in dem adversativen Nachsatz weiter behandelt wird. Anders verhält sich die AP in dem folgenden Beispiel:

(14) To mě jako nepodpoříšs?

Wirst du mich denn nicht unterstützen?

In Fragen wie in (14) wird nicht nur die bloße Information erfragt, der Sprecher fügt damit indirekt auch hinzu, dass der erfragte Sachverhalt (in diesem Falle eine Verteidigungstat) von dem Gesprächspartner erwartet wird. Gleichzeitig findet man mindestens 
in der tschechischen Version eine Andeutung darauf, dass es (doch) klar sein muss, dass es zu der besagten Tat kommen muss.

Zur Abtönung einer Aussage im Sinne und gemäß den Intentionen des Sprechers muss es nicht nur auf der Ebene des einzelnen Lexems kommen. Es können nämlich auch morphosyntaktische oder prosodische Besonderheiten entstehen, die in einigen Sprachen (wir vertreten die Auffassung, dass Tschechisch eine davon ist) die Aussage gezielt modifizieren oder dazu mindestens beitragen. Waltereit (2006: 12ff) befasst sich mit anderen Äußerungsarten der Abtönung in verschiedenen europäischen Sprachen und führt ein paar Beispiele für Kontexte an, in denen man auch ohne Partikel metasprachlich operiert. Im Französischen werden beispielsweise periphrastische Konstruktionen verwendet, in den serbo-kroatischen Sprachen kann man in Aufforderungssätzen die durch die Partikel man bewirkte Unterscheidung mithilfe des perfektiven und imperfektiven Imperativs ausdrücken, und im Englischen helfen oft geänderte Intonationskurven oder Question-Tags. Unseren Schwerpunkt werden die morphosyntaktischen Prozesse der Ausklammerung, Framing und Refrainfragen bilden.

Die Refrainfragen stellen im gesprochenen Tschechischen eine gängige Phrase dar. Obwohl es ihre Aufgabe ist, vor allem als Mittel der Vergewisserung zu dienen, kann man sie auch zu einem anderen kommunikativen Zweck nutzen; vgl.:

(15) On má zítra narozeniny? Musíš mu něco koupit, ne?!

Er hat morgen Geburtstag? Du musst ihm doch etwas kaufen!

(16) Marek je už zase nemocný.

To není žádné překvapení, on taky včera neměl na fotbale žádnou bundu, že?

Marek ist schon wieder krank.

Das ist keine Überraschung, er hatte $j a$ auch gestern beim Fußball keine Jacke an, nicht wahr?

Mit der Verwendung von Refrainfragen in beiden oben genannten Beispielen signalisiert der Sprecher dem Hörer, dass der hervorgehobene Sachverhalt nicht neu ist und als selbstverständlich zu betrachten ist. Der Fall (15) behandelt eine Situation, in der auf allgemeines Höflichkeitsverhalten hingewiesen wird. Diese Äußerung ist eher als rhetorische Frage denn als eine Vergewisserung zu deuten. Der Sprecher erinnert seinen Gesprächspartner an die Tatsache, dass man üblicherweise nicht ohne Geschenk zu einer Geburtstagsfeier geht, und er vergewissert sich beim Hörer darüber. Eine bereits bekannte Tatsache oder zumindest eine Erinnerung daran sehen wir im Beispiel (16). Hier versucht der Sprecher seinem Partner zu zeigen, dass die von ihm präsentierte Information nicht unerwartet ist, und er versucht gleichzeitig zu klären, warum, indem er auf Tatsachen hinweist, die die Situation begründen und die dem Hörer (doch) bekannt sein müssen. Sowohl diese als auch die vorangegangene Frage ist nicht als reine rhetorische Frage zu deuten. Man erwartet zwar keine direkte Antwort (im Sinne einer unmittelbar kohäsiven Replik), zugleich ist aber der Hörer gezwungen, den als selbstverständlich präsentierten Sachverhalt ausreichend zu bestätigen. 
Eine andere Erscheinung, die in der Umgangssprache häufig zu finden ist, ist die satzperiphere Positionierung einiger Ausdrücke. In Anknüpfung an Zeman (1992: 217f) und Rinas (2006a) kann man sagen, dass die abtönungskonstituierenden Mittel oft in der sog. Wackernagelposition vorkommen. Aus der Sicht der deutschen Syntaxlehre ist diese die Position am linken Rande des Mittelfeldes, in der bohemistischen Tradition operiert man eher mit den Termini präkausale, interkausale und postkausale Position. Im Folgenden werden wir aber zeigen, dass sich manche Abtönungsmitteln ausschließlich am Rande des Satzes, also außerhalb der gewöhnlichen Position, befinden. Eine davon ist das Lexem no, wie im Folgenden gezeigt wird:

(17) Zítra tam určitě bude i Jana. .. Karle?

(čte noviny) No, poslouchám.

Morgen kommt sicher auch Jana .. Karel?

(liest eine Zeitung) Ich höre ja zu.

(18) Nó, to je bašta!

Das ist aber lecker!

(19) Petr je chytrý, no.

Petr ist klug, nicht wahr.

(20) No, to je šílené!

Das ist ja Wahnsinn!

In (17) wird dem Gesprächspartner mithilfe von no angedeutet, dass ihm zugehört wird und dass man dies nicht anzweifeln muss. Sollte das Beispiel einen Nachsatz aufweisen, dann könnte er folgendermaßen realisiert werden: „Sprich weiter, ich höre zu, obwohl es vielleicht nicht so aussieht." Es ist sowohl eine betonte als auch eine unbetonte Variante möglich. In (18) äußert der Sprecher eine positive Bewertung. Es würde wahrscheinlich in einem Kontext vorkommen, wo beide Gesprächspartner dasselbe essen. Dementsprechend antizipiert man die Haltung des Gesprächspartners (oder einer Gruppe oder einer dritten Partei), die aufgrund der gemeinsamen Erfahrung die gleiche sein muss. Das gemeinsame Wissen und die Auswirkung dessen sehen wir im Beispiel (19). Die Intelligenz von Petr wird als bekannt präsentiert, und den Satz könnte man als Antwort oder Begründung deuten, die genau das von beiden Gesprächspartnern geteilte gemeinsame Wissen als Argument in sich trägt. Noch stärker ist die Evidenz der Aussage in (20) ausgedrückt. Dem Hörer wird aufgrund der Verweise auf das unterstellte Wissen angedeutet, dass die entsprechende Tatsache bestimmte (hier etwa alarmierende) Eigenschaften hat. In manchen Kontexten könnte zugleich eine widerlegende Reaktion verlangt werden.

Das Spezifikum der Beispiele (17) - (20) besteht darin, dass sie einerseits aufgrund der AP no und andererseits aufgrund ihrer festgelegten syntaktischen Struktur die ganze Aussage abtönen können. Wir haben uns entschieden, diese morphosyntaktische Erscheinung als Framing (also Einrahmen im Sinne des ganzen Satzes) anzusehen. ${ }^{4}$ Man vergleiche hierzu auch die folgenden Beispiele:

4 Nicht zu verwechseln mit der semantischen Framing-Theorie, vgl. dazu ENTMAN (1993). 
(21) *To je no hrozné.

*Petr je no chytrý.

Obwohl man im Tschechischen über keinerlei Satzrahmen spricht, wird dieses Mittel ausschließlich an den Satzrand (oder an den äußersten Rahmen der Aussage) gestellt.

Zum Schluss möchten wir noch kurz den morphosyntaktischen Prozess der Ausklammerung diskutieren. In diesem Prozess werden bestimmte Ausdrücke (vor allem Pronomina) an den Satzanfang gestellt und stellen somit die nachfolgende Information in Bezug entweder zum Gesagten oder zum Gesprächspartner. Die lexikalische Fülle dieser Ausdrücke ist teilweise verlorengegangen, ihre Funktion grammatikalisiert, und sie wird zum Zwecke der Abtönung verwendet. Am häufigsten kommt es zu diesem Prozess bei den ursprünglichen Personalpronomina on, ona, ono bzw. beim Demonstrativpronomen to:

(22) Ty s náma na hory nepojedeš?

To já pojedu, ale v sobotu už musím zase zpátky.

Du fährst mit uns nicht in die Berge?

Doch, ich werde mitfahren, aber am Samstag muss ich wieder zurück.

(23) Aneta mi zase nadává!

Ona Aneta ty nadávky nemyslí vážně.

Aneta hat wieder mit mir geschimpft!

Aneta meint es doch nicht ernst.

In (22) wird die rhematische Phrase durch die Ausklammerung mithilfe von to hervorgehoben. Es wird kommuniziert, dass die bereits besprochene Anwesenheit des Sprechers immerhin gültig ist. Gleichzeitig will der Sprecher klar sagen, dass der in der Frage enthaltene Zweifel unbegründet ist. Ähnlich wird in (23) die präsentierte Situation negiert und dem Hörer angedeutet, dass aufgrund unterstellten Wissens die besprochene Person (doch) nicht so böse sein kann, wie es dargestellt wird. In beiden Fällen sehen wir einen Appell an das bereits im Diskurs Gesagte oder auf Sachverhalte, die beiden Gesprächspartnern bekannt sind.

\section{Fazit}

In diesem Beitrag haben wir uns bemüht, einige abtönungsfähige Konstruktionen im Tschechischen zu beschreiben. Zuerst befassten wir uns mit der Klärung des Begriffs Abtönung und haben festgestellt, dass die meisten Abtönungsformen als Modifikatoren fungieren, die den Satzmodus begleiten und somit zur Einbettung der (impliziten) Sprecherintentionen in die Aussage beitragen. Für die Analyse dessen haben wir nachfolgend zwei Überprüfungsmethoden vorgestellt.

Im Rahmen der auf zwischensprachliche Äquivalenz gerichteten Analyse wurde zuerst die AP jako und die Breite ihrer kommunikativen Funktionen behandelt. Da sich aber die meisten Arbeiten im Bereich der pragmatischen Erforschung der metakommunikativen Sprache und 
dem Ausdruck der Sprechereinstellung eher den einzelnen Lexemen (überwiegend Partikeln) und dessen linguistischer Konfrontation widmen, wurde diese Auseinandersetzung auf Konstruktionen fokussiert, die, wenn nicht allein, so doch mindestens als Begleiter der Konstituierung von Abtönung im Tschechischen wirksam sind. Im Vordergrund standen dabei der Prozess der Ausklammerung (in der die rhematische Phrase mithilfe eines grammatikalisierten Pronomens hervorgehoben wird), des Framings bzw. des Einrahmens (in dem die ausschließlich satzperiphere Positionierung des Ausdrucks no und seine diversen intonatorischen Realisierungen erwähnt wurden) und die Refrainfragen (bei denen auf diskursgegebene Sachverhalte mit einer angehängten Frage hingewiesen wird). Einer der Anwendungsbereiche für die Ergebnisse dieses Beitrags könnte also die (kontrastive) Translatologie sein, denn die obengenannten tschechischen Konstruktionen sind als adäquate Übersetzungsmöglichkeiten zu dem recht breiten Repertoire der deutschen Abtönungsmittel zu betrachten.

\section{Quellen und Literatur}

Bastert, Ulrike (1985): Modalpartikel und Lexikographie: eine exemplarische Studie zur Darstellbarkeit von doch im einsprachigen Wörterbuch. Tübingen: Niemeyer.

Brausse, Ursula (1986): „Zum Problem der sogenannten Polyfunktionalität von Modalpartikeln“. In: ZPSK 39, S. 206-223.

Bublitz, Wolfram (1978): Ausdrucksweisen der Sprechereinstellung im Deutschen und Englischen. Tübingen: Max Niemeyer.

Diewald, Gabriele (1997): Grammatikalisierung. Eine Einführung in Sein und Werden grammatischer Formen. Tübingen: Niemeyer.

Gottsched, Johann Christoph (1758): Beobachtungen über den Gebrauch und Misbrauch vieler deutscher Wörter und Redensarten. Straßburg/Leipzig: Johann Amandus König.

Grepl, Miroslav/Karlík, Petr (1998): Skladba češtiny. Olomouc: Votobia.

Helbig, Gerhard (1988): Lexikon deutscher Partikeln. Leipzig: Langenscheidt Verlag Enzyklopädie.

Heynatz, Johann Friedrich (1796): Versuch eines Deutschen Antibarbarus oder Verzeichniss solcher Wörter, deren man sich in der reinen Deutschen Schreibart entweder überhaupt oder doch in gewissen Bedeutungen enthalten muss, nebst Bemerkung einiger, welcher mit Unrecht getadelt werden. Erster Band. Berlin: Verlag der königl. preuß. akadem. Kunst- und Buchhandlung.

Kolářová, Ivana (1994): „Výskyt a úloha výrazů nevím/já nevim a jako v mluvených projevech budoucích učitelů“. In: Český jazyk a literatura 45, S. 165-169.

König, Ekkehard (2010): „Dimensionen der Bedeutung und Verwendung von Modalpartikeln im Deutschen: Grundlagen einer Bestandsaufnahme. In: Harden, Theo/Hentschel, Elke (Hgg.): 40 Jahre Partikelforschung. Tübingen: Stauffenburg.

Lang, Ewald/Pasch, Renate (1988): Grammatische und kommunikative Aspekte des Satzmodus. In: Linguistische Studien 177, S. 1-24.

Masařík, Zdeněk (1982): „Vorüberlegungen zu den sog. Satzadverbien im Deutschen und Tschechischen“. In: Brünner Beiträge zur Germanistik und Nordistik 3, S. 21-35.

Meibauer, Jörg (1994): Modaler Kontrast und konzeptuelle Verschiebung: Studien zur Syntax und Semantik deutscher Modalpartikeln. Tübingen: Niemeyer.

Nekula, Marek (1996): System der Partikeln im Deutschen und Tschechischen: Unter besonderer Berücksichtigung der Abtönungspartikeln. Tübingen: Max Niemeyer Verlag. 
Rinas, Karsten (2006a): „Abtönungspartikeln, das Mittelfeld und die Wackernagelposition“. In: brücken. Germanistisches Jahrbuch Tschechien - Slowakei 2006. Bonn: DAAD.

Rinas, Karsten (2006b): Die Abtönungspartikeln doch und ja: Semantik, Idiomatisierung, Kombinationen, tschechische Äquivalente. Frankfurt am Main - Berlin - Bern - Bruxelles - New York - Oxford - Wien: Peter Lang.

Rinas, Karsten (2011): Sprache, Stil und starke Sprüche. Bastian Sick und seine Kritiker. Darmstadt: Wissenschaftliche Buchgesellschaft.

Rinas, Karsten (2013): „Tři „nové modifikační částice v češtině: schválně, normálně a jako“. In: Slovo a slovesnost, 74. S. 163-177.

Spěváková, Dana (1992): „Zu einigen Übersetzungsmöglichkeiten der deutschen Abtönungspartikeln ins Tschechische." In: Brünner Beiträge zur Germanistik und Nordistik 8, S. 43-51.

Thurmair, Maria (1989): Modalpartikeln und ihre Kombinationen. Tübingen: Max Niemayer Verlag.

Waltereit, Richard (2006): Abtönung. Zur Pragmatik und historischen Semantik von Modalpartikeln und ihren funktionalen Äquivalenten in romanischen Sprachen. Tübingen: Max Niemayer Verlag.

Weydt, Harald (1969): Abtönungspartikel. Die deutschen Modalwörter und ihre französischen Entsprechungen. Bad Homburg: Gehlen.

Zeman, Jaromír (1992): „Wortstellungsschemata im Deutschen und im Tschechischen - Versuch einer vergleichenden Darstellung." In: Germanistentreffen Bundesrepublik Deutschland - ČSFR, 6. - 10. 10. 1992. Dokumentation der Tagungsbeiträge. Bonn: DAAD, S. 217-234.

Mgr. Jan Ciosk / ciosk.jan@gmail.com

Univerzita Palackého v Olomouci, Filozofická fakulta, Katedra germanistiky

Křižkovského 10, 77900 Olomouc, Česká republika

This work can be used in accordance with the Creative Commons BY-SA 4.0 International license terms and conditions (https://creativecommons.org/licenses/by-sa/4.0/legalcode). This does not apply to works or elements (such as image or photographs) that are used in the work under a contractual license or exception or limitation to relevant rights 
